This item was submitted to Loughborough's Research Repository by the author.

Items in Figshare are protected by copyright, with all rights reserved, unless otherwise indicated.

\title{
Software applications in filter control, data acquisition and data analysis
}

PLEASE CITE THE PUBLISHED VERSION

PUBLISHER

(C) Elsevier

VERSION

AM (Accepted Manuscript)

LICENCE

CC BY-NC-ND 4.0

REPOSITORY RECORD

Tarleton, E.S., and Richard J. Wakeman. 2009. "Software Applications in Filter Control, Data Acquisition and Data Analysis”. figshare. https://hdl.handle.net/2134/4918. 
This item was submitted to Loughborough's Institutional Repository (https://dspace.lboro.ac.uk/) by the author and is made available under the following Creative Commons Licence conditions.

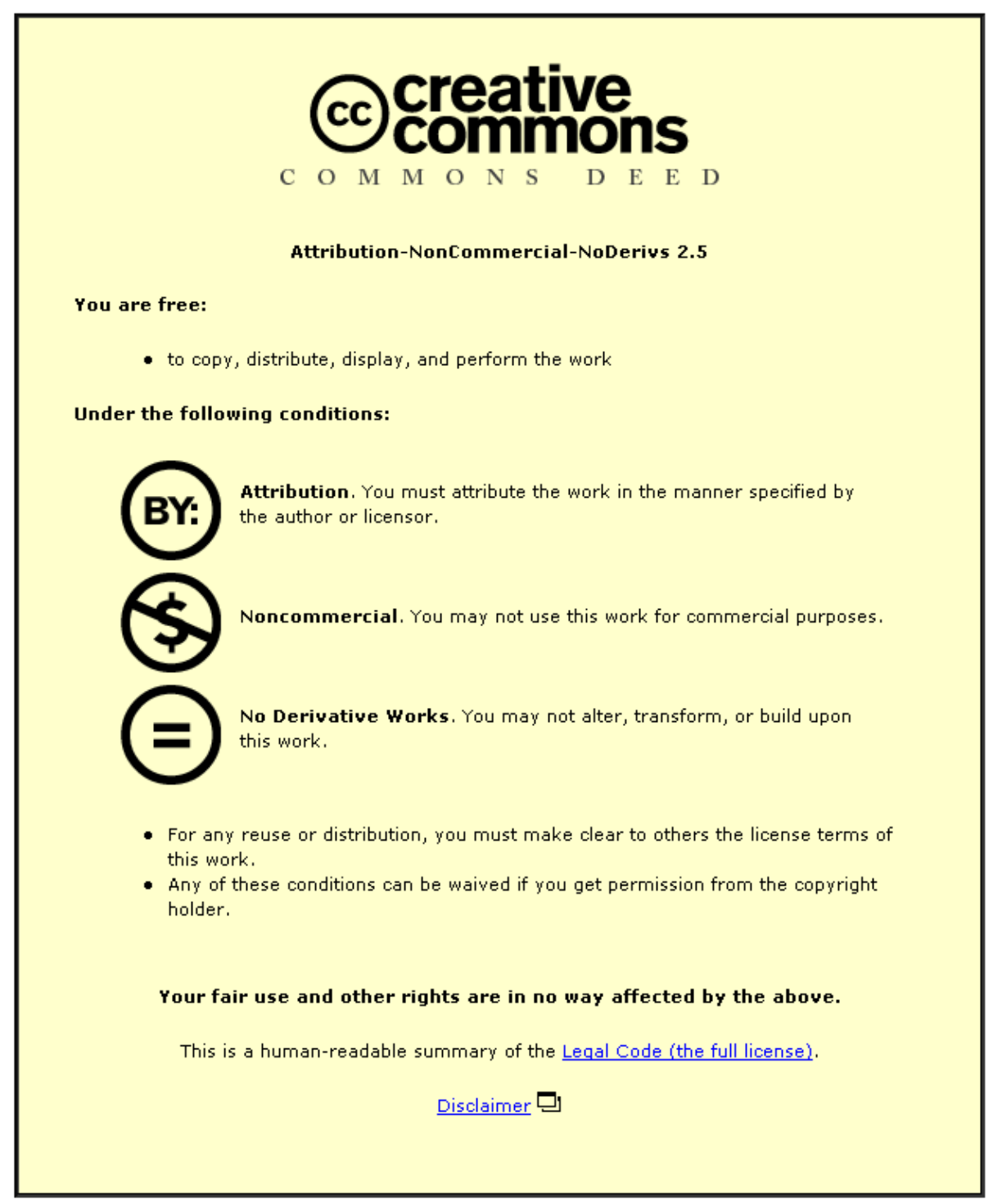

For the full text of this licence, please go to: http://creativecommons.org/licenses/by-nc-nd/2.5/ 


\title{
SOFTWARE APPLICATIONS IN FILTER CONTROL, DATA ACQUISITION AND DATA ANALYSIS
}

\author{
E.S. Tarleton (e.s.tarleton@lboro.ac.uk) and R.J. Wakeman \\ Department of Chemical Engineering, Loughborough University, Loughborough, Leics., LE11 3TU, \\ UK.
}

\begin{abstract}
This paper describes aspects of computer software use in filter apparatus control and data acquisition and details a robust software procedure for analysing cake filtration and consolidation data. It is shown how software can be used to automate state-of-the-art laboratory scale leaf filters and piston presses and facilitate the real-time display of parameters such as liquor transport rates and transient cake solids concentrations. Typical expression data from the piston press apparatus are used to illustrate an interactive software analysis procedure which uses basic experimental information provided by the operator to calculate parameters which characterise a separation; these include the specific cake resistance, voids ratio and consolidation coefficient. Methods of overcoming the problems of data scatter are highlighted along with the procedures used to provide expert guidance during data analysis. The filtration and consolidation theories which underpin the software are also briefly described to show how scale-up constants can be software derived for subsequent use in process simulations.
\end{abstract}

\section{KEYWORDS}

Cake filtration; Consolidation; Software; Data analysis; Automation

\section{INTRODUCTION}

Of the unit operations available to the process engineer, those associated with solid/liquid separation are perhaps the most difficult to specify. Although a robust equipment selection procedure now exists ${ }^{1}$ and detailed filter design procedures are becoming available ${ }^{2}$, our lack of fundamental understanding for suspension behaviour necessitates recourse to laboratory and/or pilot plant tests in order to establish parameters which characterise a separation; typical apparati are either a leaf filter or piston press. The difficulties associated with reliable data acquisition in filtration experiments are well known and present considerable problems to the separation technologist who is required to specify filtration plant. Whilst theories for analysing cake filtration and consolidation are both well established ${ }^{2-4}$, their application is often ill-defined and this creates further difficulties as variations in procedures inevitably lead to errors.

The current work presents a philosophy which aims to provide a co-ordinated approach to filter experimentation and data analysis. It is argued that well instrumented and computer software automated apparatus in conjunction with consistent software aided analysis procedures allows the best opportunity to acquire sufficient and accurate data for the correct simulation and sizing of process scale filters.

\section{APPARATUS CONTROL AND DATA ACQUISITION}

The schematic in Figure 1 shows a state-of-the-art, laboratory scale apparatus capable of automated data acquisition during filtration, washing and deliquoring phases of the filter cycle. The level of hardware and software sophistication is varied according to requirements ${ }^{2,5-7}$. 
The basic hardware comprises of a stainless steel (s/s) dead-end Nutsche filter (area 5 to $500 \mathrm{~cm}^{2}$ ) and two s/s storage vessels connected by s/s piping and computer controlled electro-pneumatic valving. The storage vessels incorporate temperature sensors and stirrers and contain the feed suspension and wash water as appropriate. The filter cell can be rotated to the desired orientation to allow filtration onto upward, downward or sideways facing filter surfaces. A heater/cooler system regulates the temperature of the filter cell and storage vessels by continuously passing a fluid through their surrounding jackets to facilitate operation over the range 0 to $70^{\circ} \mathrm{C}$.

Measurement transducers attached to the apparatus allow pressures and other parameters to be recorded and/or controlled by the interfaced personal computer and dedicated software.

The pressures required to progress filtrations are provided by a compressor and an electronic pressure regulator over the range $0-1 \mathrm{MPa}$. The regulator is software adjusted by the computer and the filtrate flow rate is semi-continuously transmitted to the computer via successive timed readings of mass from an electronic balance. By monitoring the flow rate in such a manner, use of a software control algorithm allows either constant pressure, constant rate or variable pressure/variable rate filtration to be performed without changing the suspension properties through inappropriate pumping operations; the control algorithm is based on the general form:

$p=p_{s}+K\left(\varepsilon+\frac{1}{T_{i}} \int_{0}^{t} \varepsilon d t+T_{d} \frac{d \varepsilon}{d t}\right)$

where $p$ is the required pressure, $p_{s}$ is a constant, $K$ the system gain, $\varepsilon$ the flow error (i.e. required flow - measured flow), $t$ is time, $T_{i}$ the integral time and $T_{d}$ the derivative time. The rate and magnitude of the pressure adjustments is dependent on the nature of the feed, the compressibility of the filter cake and the desired process conditions.

The real time monitoring of cake growth rate and transient changes in filter cake structure during filtration (and if required solute concentration profiles in the cake during washing and/or cake saturation profiles during deliquoring) is provided by series of small electrodes fitted internally within the filter cell. The electrodes which generally protrude a short distance into the cell are arranged in single vertical planes or, if maximum data are required, in sequences of horizontal rings. Signals to electrode pairs are switched by the attached computer via software and electronic circuitry to facilitate measurements.

Figure 2 shows a schematic of an automated state-of-the-art piston press which facilitates both filtration and consolidation at pressures up to $40 \mathrm{MPa}$. An otherwise conventional piston press apparatus is augmented with the addition of a personal computer and a software driven pressure regulator which allows the pressure applied to the piston to be varied according to a pre-set pattern (e.g. constant, ramp, sinusoid etc.). The interfaced rotary encoder, force transducer and electronic balance respectively allow semi-continuous measurements of piston displacement, transmitted pressure and expressed liquid mass which are interpreted and displayed via computer software. Where maximum information is required the piston can also be rotated using software control and a suitable mechanical gearbox to impart shear as well as compressive forces to the filtering and/or consolidating solid/liquid mixture; such measurements are useful for determining performance on, for instance, belt press filters.

The use of computer software control allows data to be acquired in a repeatable and reliable manner with a minimum of operator interference. The real time measurement of experimental parameters also allows the continuous display of results and Figure 3 shows an example. Although the equipment shown in Figures 1 and 2 probably represents the limits of what can be achieved with readily available technology, such apparatus are yet to be used widely for filter data acquisition. They do, however, offer the basis from which to develop standard apparatus and methods for filtration testing and their inherent flexibility facilitates integration with software analysis techniques. 


\section{SOFTWARE DATA ANALYSIS}

The analysis of sequential filtration and consolidation phases in, for instance, a piston press expression test requires that sufficient basic experimental data be acquired for each phase to allow the co-ordinated use two modelling theories.

\section{Basic Modelling Theory}

With our current state of knowledge it is usual to characterise macroscopic cake filtration behaviour through the use of four empirical scale-up constants $\left(a_{0}, n,\left(e_{0}\right)_{f}\right.$ and $\left.b_{f}\right)$ such that specific cake resistance $(\alpha)$ and a measure of cake structure such as voids ratio $(e)$ are related for practical purposes to the filtration pressure $\left(\Delta p_{f}\right)$ by:

$$
\begin{aligned}
& \alpha_{a v}=\alpha_{0}(1-n) \Delta p_{f}^{n} \\
& \left(e_{a v}\right)_{f}=\left(e_{0}\right)_{f}-b_{f} \log \left(\Delta p_{f}\right)
\end{aligned}
$$

The conventional way to derive values for the scale-up constants is to perform series of filtration experiments at a range of constant pressures and analyse the resultant data using the general filtration equation where the filtration time $\left(t_{f}\right)$ and cumulative volume of filtrate $\left(V_{f}\right)$ are related by:

$$
\frac{t_{f}}{V_{f}}=\frac{\alpha_{a v} \mu \rho s}{2 A^{2} \Delta p_{f}\left(1-s\left(1+\left(e_{a v}\right)_{f}\left(\rho / \rho_{s}\right)\right)\right)} V_{f}+\frac{\mu R_{m}}{A \Delta p_{f}}
$$

where $\mu$ is the filtrate viscosity, $s$ the solids mass fraction in the feed, $A$ the filter area, $\rho$ and $\rho_{s}$ the filtrate and solids densities respectively and $R_{m}$ the filter medium resistance. When necessary, the changing cake thickness $(h)$ can also be theoretically related to the volume of filtrate by:

$$
h=\frac{V_{f}\left(1+e_{a v}\right)}{A\left(\frac{\rho_{s}}{\rho}\left(\frac{1}{s}-1\right)-e_{a v}\right)}
$$

The cake consolidation process is also conventionally described by series of constant pressure experiments and empirical relations such that the consolidation coefficient $\left(C_{c}\right)$ and ultimate voids ratio $\left(\left(e_{a v}\right)_{c}\right)$ are related to the time $\left(t_{c}\right)$ and consolidation pressure $\left(\Delta p_{c}\right)$ by five scale-up constants $\left(C_{e 0}, \gamma,\left(e_{0}\right)_{c}, b_{c}\right.$ and $\left.v\right)$ such that:

$$
C_{c}=C_{e 0} \Delta p_{c}^{r}
$$

$\left(e_{a v}\right)_{c}=\left(e_{0}\right)_{c}-b_{c} \log \left(\Delta p_{c}\right)$

$U_{c}=\frac{h_{t r}-h}{h_{t r}-h_{\infty}}=\left(\sqrt{\frac{4 T_{c}}{\pi}}\right)\left(1+\left(\sqrt{\frac{4 T_{c}}{\pi}}\right)^{2 v}\right)^{-(1 / 2 v)}$

$$
T_{c}=\frac{i^{2} C_{c} t_{c}}{\omega_{0}^{2}}
$$


where $U_{c}$ is the consolidation ratio, $h_{t r}$ the cake thickness at the transition from filtration to consolidation, $\mathrm{h}_{\infty}$ the ultimate cake thickness, $T_{c}$ the dimensionless consolidation time, $i$ the number of filter surfaces (normally unity in a laboratory test) and $\omega_{0}$ the volume of cake solids per unit filter area. For small values of $T_{c}$, eqn. (8) reduces to:

$U_{c} \approx \sqrt{\frac{4 T_{c}}{\pi}}=C_{1} \sqrt{t_{c}}$

where $C_{1}$ is a constant obtained by combination with eqn. (9) and equal to $\left(2 i / \omega_{0}\right) \sqrt{C_{c} / \pi}$.

When experimental data have been reliably acquired, using for instance the automated apparatus shown in Figures 1 and 2, eqns. (2)-(10) provide a method of evaluating the parameters required for process filter calculations and sizing. Such data and equations, however, are of maximum benefit when combined with robust data analysis and scale-up procedures. In the opinion of the authors, data analysis is best dealt with by dedicated personal computer software which interacts easily with the user to provide expert assistance in data interpretation whilst avoiding the psychological barriers often associated with computer use. Such analysis software, which forms part of a program suite for selection, analysis and filter simulation, has been under development by the authors for some time and its main features are described in the remainder of this paper.

\section{Software Analysis of an Experiment}

The first step in the analysis of an individual (constant pressure) experiment requires basic data entry to the computer software. This is an interactive process whereby the user types known numerical values from the leaf filter or piston press experiment. The required values include individual properties of the solids and liquids forming the test suspension (such as density), process conditions (such as applied pressure) and a sequence of data pairs which record and quantify the progress of an experiment. The latter may comprise time vs. cumulative filtrate volume or cake height or piston displacement as appropriate and conversions from one data form to another can be readily performed by software using, for example, eqn. (5). The test data are transformed by software algorithm and data of the forms shown schematically in Figure 4 are typically produced. The $t / V$ vs. $V$ and $d t / d V$ vs. $V$ plots are well known in constant pressure filtration analysis and in accordance with forms of the general filtration equation (e.g. eqn. (4)) the initial linear portion represents the extent of the filtration phase. The relatively sharp change in gradient towards the end of a test is also frequently observed and arises as a consequence of a gas deliquoring phase (in a batch leaf filter experiment) or a consolidation phase (in the case of a typical piston press expression experiment). The identification of the transition between phases is important for subsequent data analysis and it is necessary to provide for robust software algorithms which are capable of dealing with natural data scatter.

Although $t / V$ vs. $V$ data are used in the calculation procedures, progressive development and testing with a wide range of laboratory experimental data have shown that the plots of $|d h / d \sqrt{t}|$ vs. $t$ (designated Method 1 ) and $V$ vs. $\sqrt{t}$ (designated Method 2) are the most appropriate for representing the software identification of a transition point. Both methods employ an approximation to eqn. (4), whereby $R_{m}$ is assumed to be negligible and:

$$
t \approx \frac{\alpha_{a v} \mu \rho s}{2 A^{2} \Delta p\left(1-s\left(1+\left(e_{a v}\right)_{f}\left(\rho / \rho_{s}\right)\right)\right)} V^{2}=C_{2} V^{2}
$$

where $C_{2}$ is a constant. Manipulation of eqn. (11) gives the governing equation for Method 2: 
which indicates a data linearity over the filtration phase. Differentiating eqn. (11) w.r.t. $t$, combining with eqn. (5) and rearranging gives the governing equation for Method 1:

$\frac{d V}{d \sqrt{t}} \propto \frac{d h}{d \sqrt{t}}=$ constant

which suggests that values of $|d h / d \sqrt{t}|$ remain constant with time over the filtration phase.

Using the piston press expression data shown in Table 1 as an illustrative example, Figures 5 and 6 give representations of the two graphs which are successively presented to the software user. The first, a plot of $V$ vs. $\sqrt{t}$, is employed purely as an alternative illustration of the position for the transition from filtration to consolidation as predicted by Method 2. To identify the transition, the software utilises the property of ideal linearity during the filtration phase and performs a series of least square regressions. These commence from an initial choice of three $V$ vs. $\sqrt{t}$ data pairs at a smaller elapsed time and finish with a calculation using all the available data pairs; the gradient of the regressed line being recorded in each case. The sequence of gradients is examined by the software and the time $\left(t_{T}\right)$ corresponding to the phase transition is identified from a position in the dataset (designated $(\sqrt{t})_{n 1}$ ) where three successive falls in gradient are recorded such that $t_{T}=0.5\left((t)_{n 1-4}+(t)_{n 1-3}\right)$ where $n 1$ is a counter.

Following the visual presentation of Figure 5, the computer display is refreshed with a log-log plot of $|d h / d \sqrt{t}|$ vs. $t$ which includes two interactive/user moveable cursors and an indication of the transition point as estimated by both Methods 1 and 2 (see Figure 6). Whilst experience has shown this form of plot to generally give a sharp indication of phase transition, reliable software interpretation has proved difficult due to the often unpredictable influence of even relatively minor data scatter on $d h / d \sqrt{t}$ values and the plot is essentially a visual representation for the user. The second estimate for the transition (i.e. by Method 1 ) is provided by the software through simultaneous numerical evaluations on the $t / V$ vs. $V$ and $U_{c}$ vs. $\sqrt{t}$ datasets. The estimation method, which is not displayed, relies upon the calculation of four gradients $\left(G_{1}-G_{4}\right)$ as illustrated in Figures 7 and 8 . Up to the correct transition point a $t / V$ vs. $V$ plot maintains a constant gradient in accordance with eqn. (4) and immediately after the transition the $U_{c} v s . \sqrt{t}$ plot also shows linearity in accordance with eqn. (10). An incorrect choice of the transition between the filtration and consolidation phases causes excessive deviations on one or both plots. Thus, by performing repeated calculations where a fresh estimate of the transition is made each time, it is possible to form a sequence of values for the sum of the ratios $\left(G_{S}\right)$ such that:

$G_{S}=\left(\right.$ maximum of $\frac{G_{1}}{G_{2}}$ and $\left.\frac{G_{2}}{G_{1}}\right)+\left(\right.$ maximum of $\frac{G_{3}}{G_{4}}$ and $\left.\frac{G_{4}}{G_{3}}\right)$

and identify the transition to occur when $G_{S}$ takes a value closest to 2 . Whilst the phase transitions suggested by Methods 1 and 2 are reliable in the majority of instances, such calculation procedures are not infallible and the two interactive cursors represented on Figure 6 allow the user to override a suggested transition as desired. The datapoints positioned between the two vertical cursor lines represent the extent of the filtration phase. 
With the transition defined by the user, plots of the form shown in Figures 7 and 8 are now sequentially displayed on the computer screen. If the user is dissatisfied with the form of either plot then the transition can be redefined via a looping process which commences with the redisplay of Figure 5 and includes indications of the previously identified transition. When the user is satisfied with the chosen transition, the software uses linear regression to calculate the gradient of the $t / V$ vs. $V$ dataset for the filtration phase and the rest of the typed experimental data is used to determine characteristic parameters for the filtration phase (see Table 2).

At this stage the analysis of the filtration phase is complete and provided there are sufficient data available, a graph of $U_{c}$ vs. $\sqrt{T_{c}}$ (Figure 9 ) is displayed to the user to commence analysis of the consolidation phase; the consolidation time is assumed to start at the time $t_{T}$. According to eqns. (8) and (10) the initial portion of the plot is linear and analysis requires that the extent of the linear portion be defined. The software provides a suggestion by employing linear regression. A calculation sequence is invoked which evaluates the regression correlation coefficient for a progressively increasing number of $U_{c}$ vs. $\sqrt{T_{c}}$ datapoints; the minimum number being the first three datapoints and the maximum including all the datapoints. The number of points giving the highest correlation coefficient is marked as the suggested extent of the linear portion. The user again has the opportunity to override the software choice by movement of a single interactive cursor. When the correct position has been identified, the gradient of the regression fit over the linear portion is used in eqns. (9) and (10) to evaluate the consolidation coefficient $\left(C_{c}\right)$. A final regression analysis is now performed to provide a fit of eqn. (8) to all of the $U_{c}$ vs. $\sqrt{T_{c}}$ data using $v$ as the curvefit parameter (see Figure 10). Values of $v$ over the range $0.5-5$ are sequentially substituted by the software (without display to the user) and in each case the variance between the theoretical predictions given by eqn. (8) and the experimental data is determined. By progressive refinement of the range over which $v$ is varied, the value of $v$ giving the minimum variance can be established.

With all the calculations complete, the numerical results are presented to the user in tabular form (see Table 2), whence the user has the option to print a data sheet of results, display results graphically and/or save the data to disk for further analysis and evaluation of scale-up constants.

\section{Software Determination of Scale-up Constants}

The software analysis of an individual leaf filter or piston press experiment provides a consistent procedure for the calculation of $\alpha_{a v}$ and $\left(e_{a v}\right)_{f}$ for the filtration phase and where appropriate $\left(e_{a v}\right)_{c}$, $C_{c}$ and $v$ for a consolidation phase. The first four of these parameters are associated with eight scale-up constants in accordance with eqns. (2), (3), (6) and (7) and sequences of experiments at different constant pressures can be analysed using the software described to facilitate their calculation.

Provided the results for each experiment analysis are recorded to computer disk, a further interactive aspect of the software allows the user to type a list of filenames which correspond with these analyses and then invoke an automated procedure. Values of $\alpha_{a v},\left(e_{a v}\right)_{f},\left(e_{a v}\right)_{c}$ and $C_{c}$ are read from the disk file for each analysed experiment, correlated with the associated $\Delta p$ values using linear regression and the results are visually displayed to the user in both graphical and tabular forms (Table 3 shows a typical example). The regression correlation coefficient is displayed in each case and the user has the further option of defining a more specific region over which to perform data correlation using interactive cursors.

\section{CONCLUSIONS}


The degree to which software is used to control and monitor filter apparatus, perform data analysis and facilitate filter simulation is likely to grow in future years as the technology becomes more accessible. The advantages that software provides are widespread, although yet to be fully exploited. There seems to be scope to develop software for many applications in solid/liquid separation and the work presented in this paper regarding data analysis merely provides a basis from which to progress. With the emergence of comprehensive filter simulations ${ }^{2,8-10}$ we are perhaps entering a phase in filtration technology which will move our knowledge away from an over reliance on heuristics and toward widely recognised, accepted and robust filtration philosophies.

\section{NOMENCLATURE}

$\begin{array}{ll}A & \text { filter area }\left(\mathrm{m}^{2}\right) \\ b & \text { constant } \\ C_{1}, C_{2} & \text { constants (various units) } \\ C_{c} & \text { modified consolidation coefficient }\left(\mathrm{m}^{2} \mathrm{~s}^{-1}\right) \\ C_{e 0} & \text { modified consolidation coefficient at unit applied pressure }\left(\mathrm{m}^{2} \mathrm{~s}^{-1} \mathrm{~Pa}^{-\gamma}\right) \\ e_{0} & \text { voids ratio at unit applied pressure } \\ G_{1}-G_{4} & \text { gradients on filtration and consolidation plots (various units) } \\ G_{S} & \text { sum of the ratio of gradients } \\ h & \text { cake thickness (m) } \\ h_{t r} & \text { cake thickness at the transition from filtration to consolidation (m) } \\ h_{\infty} & \text { ultimate cake height }(\mathrm{m}) \\ i & \text { number of filter surfaces } \\ K & \text { apparatus gain } \\ n & \text { compressibility index } \\ n 1 & \text { counter in a data sequence } \\ p & \text { required pressure (Pa) } \\ p_{s} & \text { constant (Pa) } \\ \Delta p & \text { pressure difference (Pa) } \\ R_{m} & \text { filter medium resistance }\left(\mathrm{m}^{-1}\right) \\ s & \text { mass fraction of solids in feed suspension } \\ t & \text { time (s) } \\ t_{T} & \text { time at the transition between filtration and consolidation }(\mathrm{s}) \\ T_{c} & \text { dimensionless consolidation time } \\ U_{c} & \text { consolidation ratio } \\ V & \text { cumulative volume of filtrate (m } \\ x & \text { particle size (m) }\end{array}$

\section{Greek symbols}

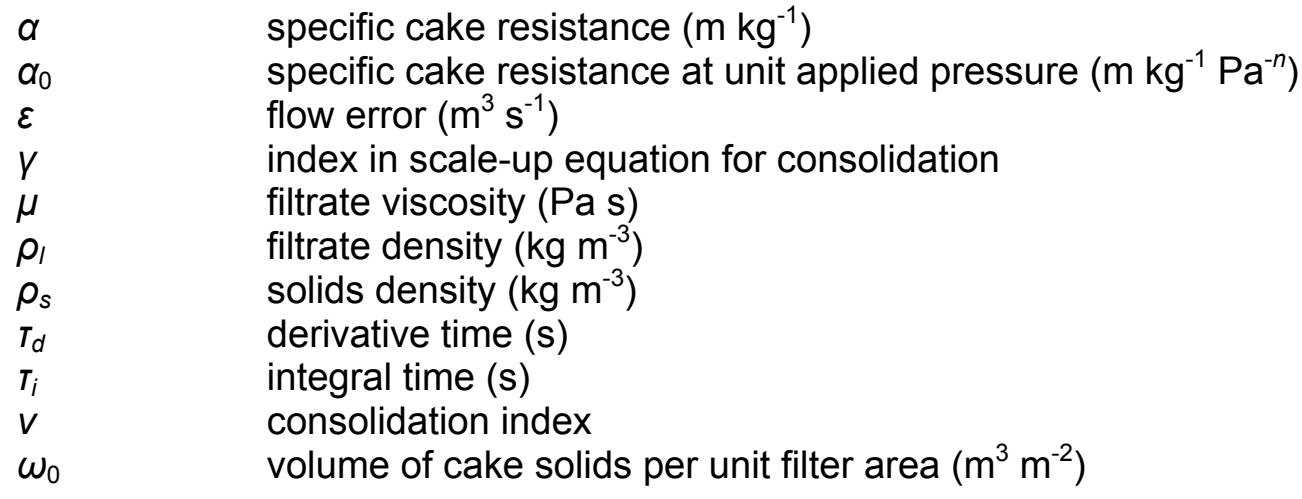

\section{Subscripts and superscripts}




$\begin{array}{ll}f & \text { filtration phase } \\ c & \text { consolidation phase } \\ a v & \text { average value }\end{array}$

\section{REFERENCES}

1. Tarleton E.S. and Wakeman R.J., 1991, Solid/Liquid Separation Equipment Selection and Design: $p^{c}$-SELECT - Personal computer software for the analysis of filtration and sedimentation test data and the selection of solid/liquid separation equipment, Separations Technology Associates, Loughborough.

2. Wakeman R.J. and Tarleton E.S., 1999, Filtration: Equipment Selection, Modelling and Process Simulation, 450 pages, Elsevier, Oxford.

3. Shirato M., Murase T. and Iwata M., 1986, Deliquoring by expression - theory and practice, in Progress in Filtration and Separation 4, Wakeman R.J. (Ed.), pp.181-287, Elsevier, Amsterdam.

4. Wakeman R.J., Sabri M.N. and Tarleton E.S., 1991, Factors affecting the formation and properties of wet compacts, Powder Technol., 65, 283.

5. Tarleton E.S. and Hancock D.L., 1996, The imaging of filter cakes through electrical impedance tomography, Filt. and Sepn., 33(6), 491.

6. Tarleton E.S. and Hancock D.L., 1997, Using mechatronics for the interpretation and modelling of the pressure filter cycle, Trans IChemE, 75(A), 298.

7. Tarleton E.S., 1998, A new approach to variable pressure filtration, Min. Engng., 11(1), 53.

8. Wakeman R.J. and Tarleton E.S., 1994, A framework methodology for the simulation and sizing of diaphragm filter presses, Min. Engng., 7(11), 1411.

9. Wakeman R.J. and Tarleton E.S., 1990, Simulation and process design of the filter cycle, Filt. and Sepn., 27(6), 412.

10. Tarleton E.S. and Wakeman R.J., 1994, Simulation, modelling and sizing of pressure filters, Filt. and Sepn., 31(4), 393 
FIGURES AND TABLES

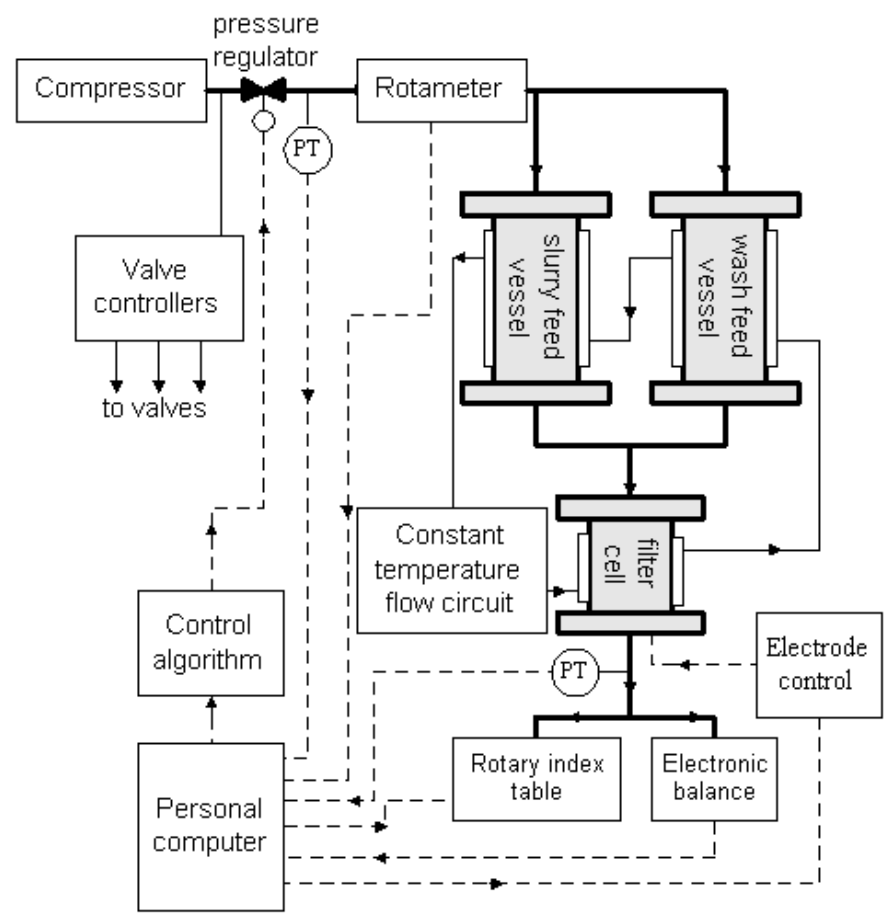

Figure 1: Schematic of an automated leaf filter apparatus for investigating filtration, washing and deliquoring processes.

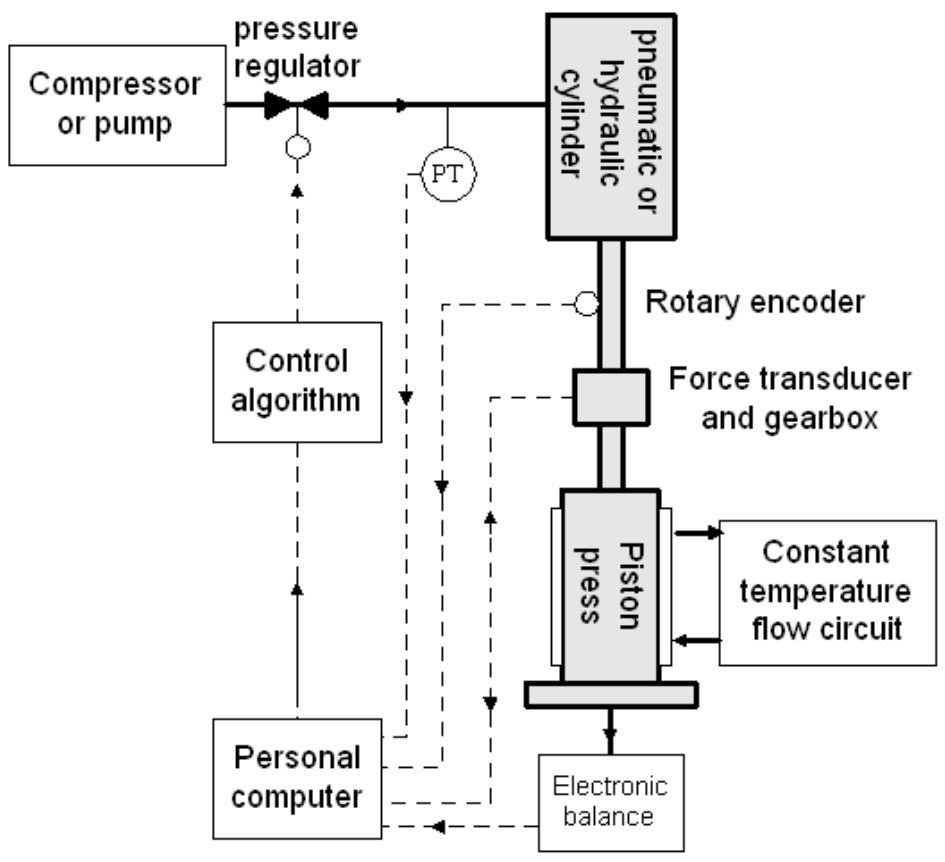

Figure 2: Schematic of an automated piston press apparatus for investigating filtration and consolidation processes. 


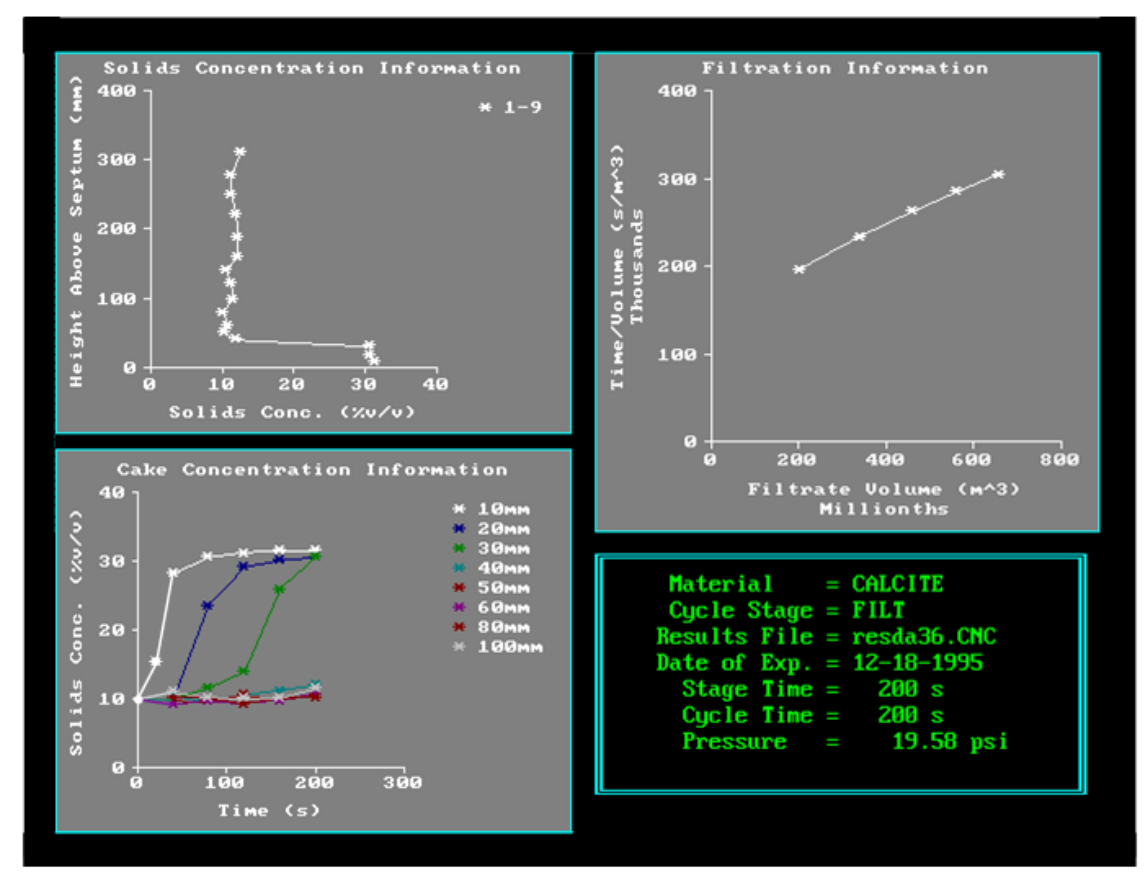

Figure 3: An example of the real-time computer display shown during operation of the apparatus in Figure 1. 

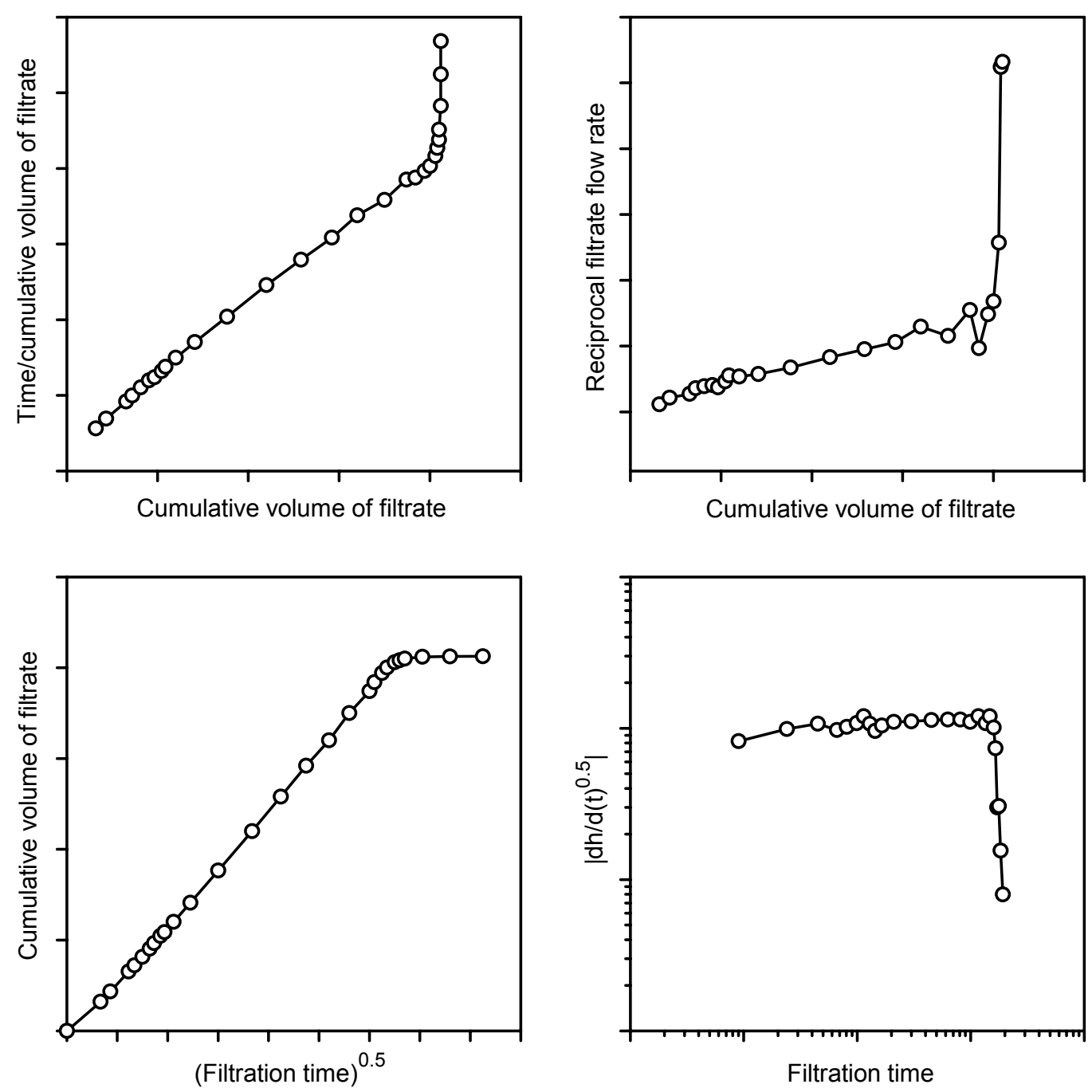

Filtration time

Figure 4: Schematic of typical data forms which indicate transition from a filtration phase. 


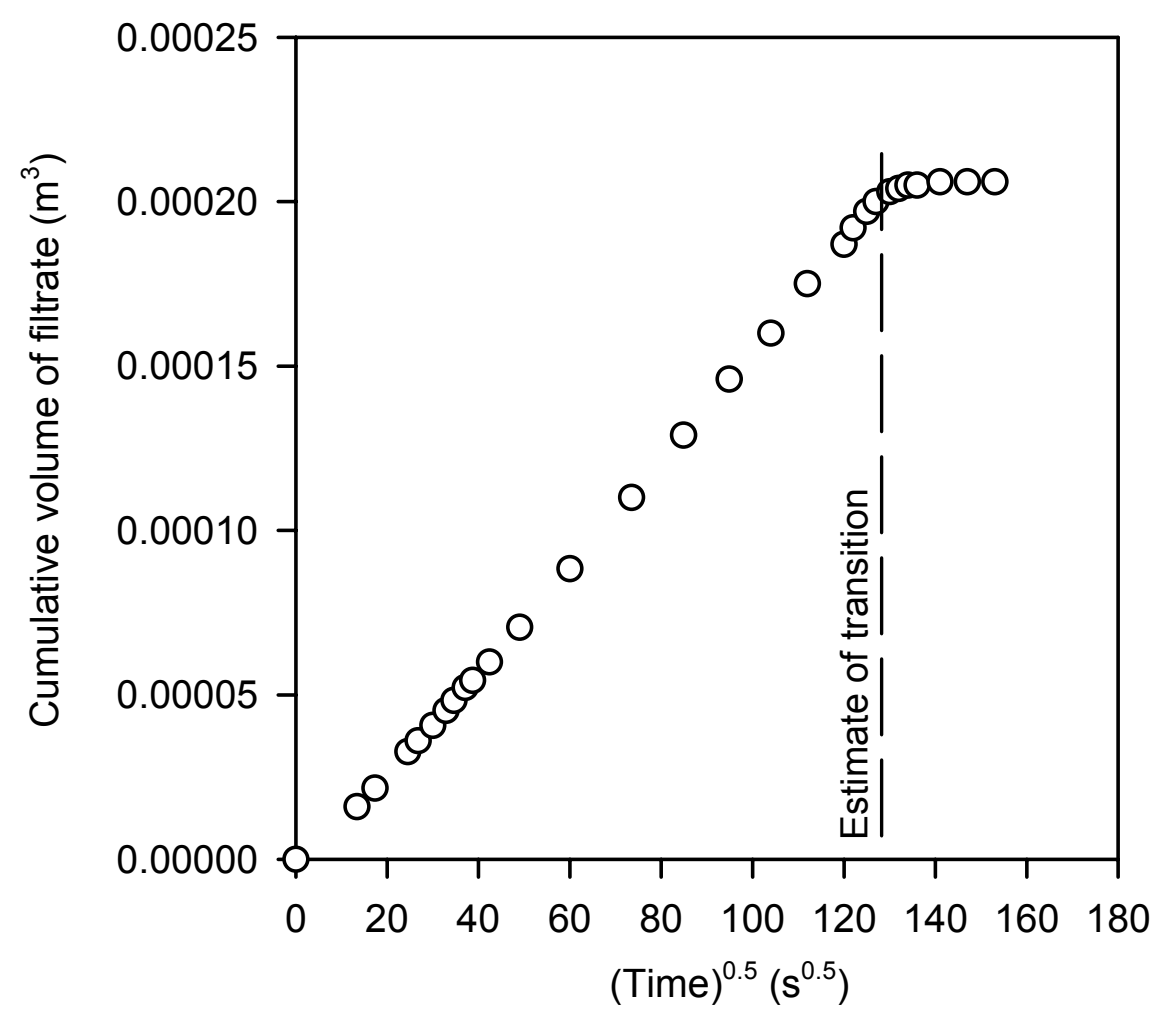

Figure 5: Illustration of the transition from filtration to consolidation as predicted by Method 2.

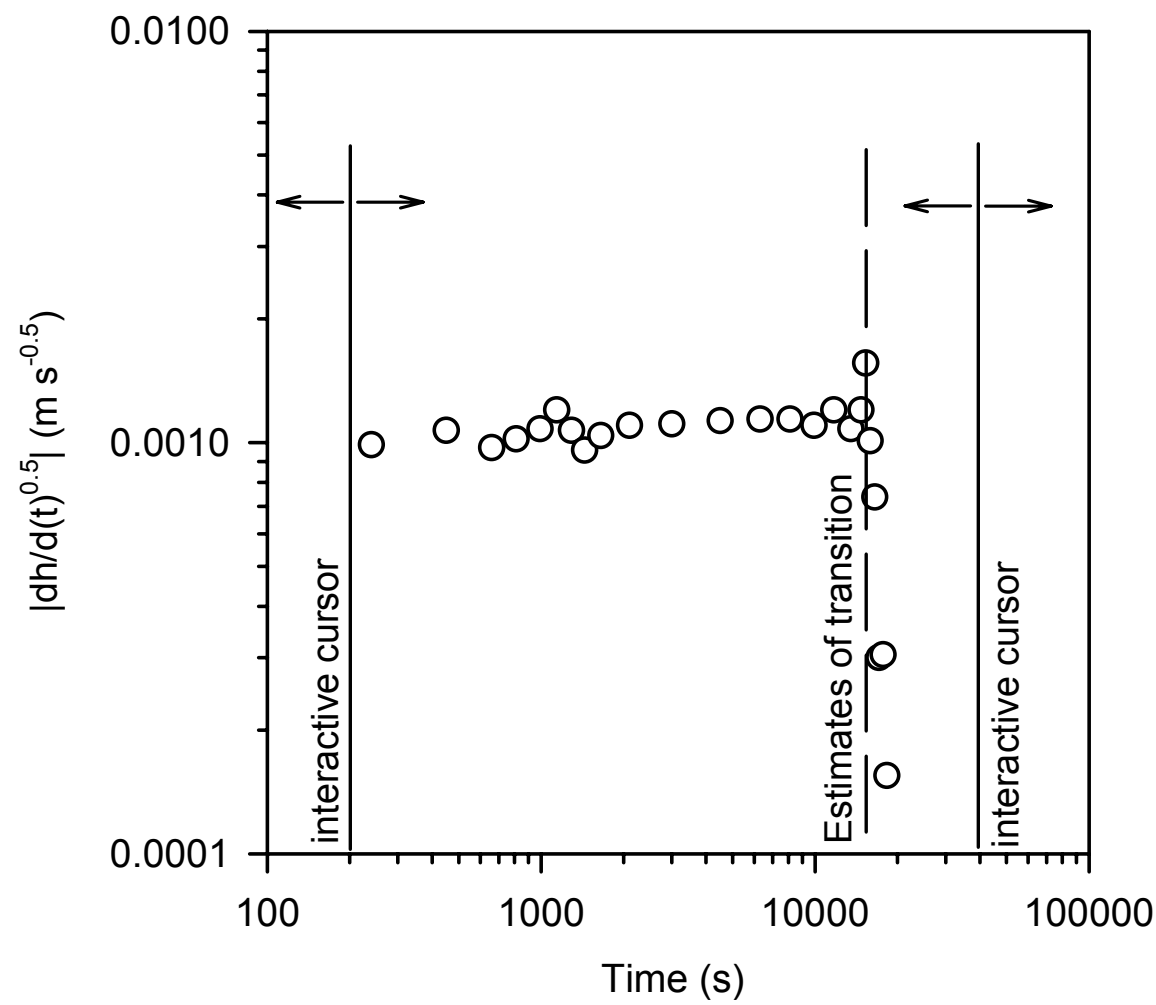

Figure 6: Plot used to display the estimated transitions filtration to consolidation. 


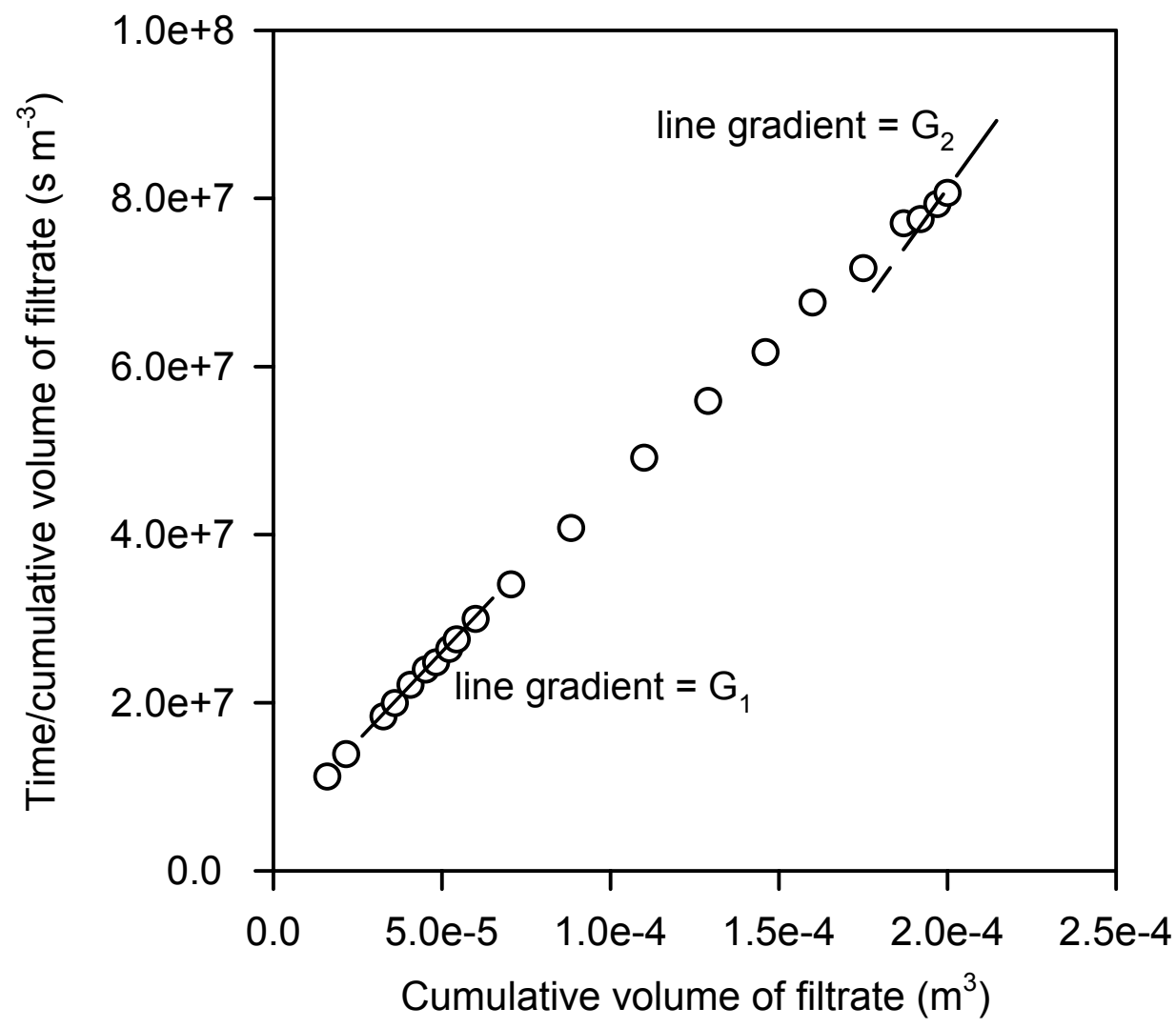

Figure 7: Form of the data used to identify the filtration phase by Method 1.

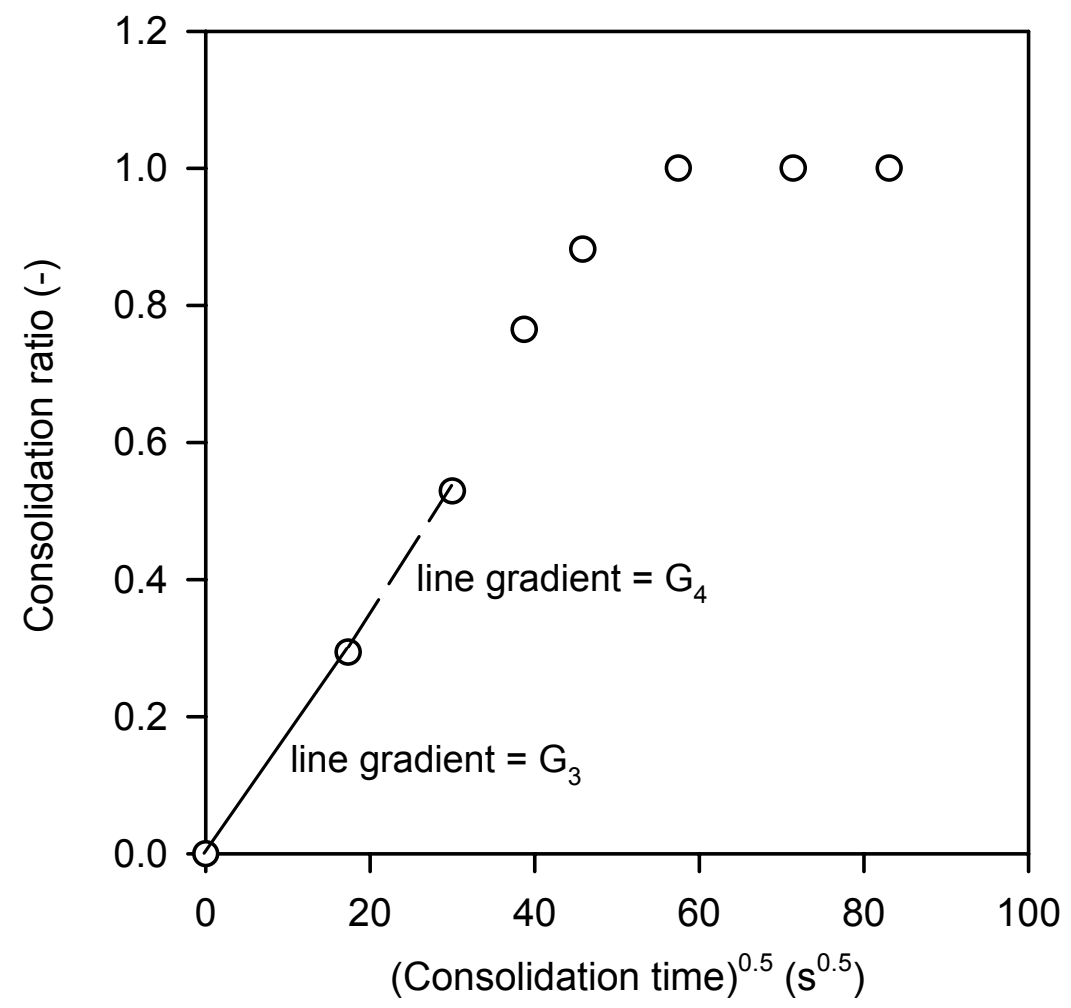

Figure 8: Form of the data used to identify the consolidation phase by Method 1. 


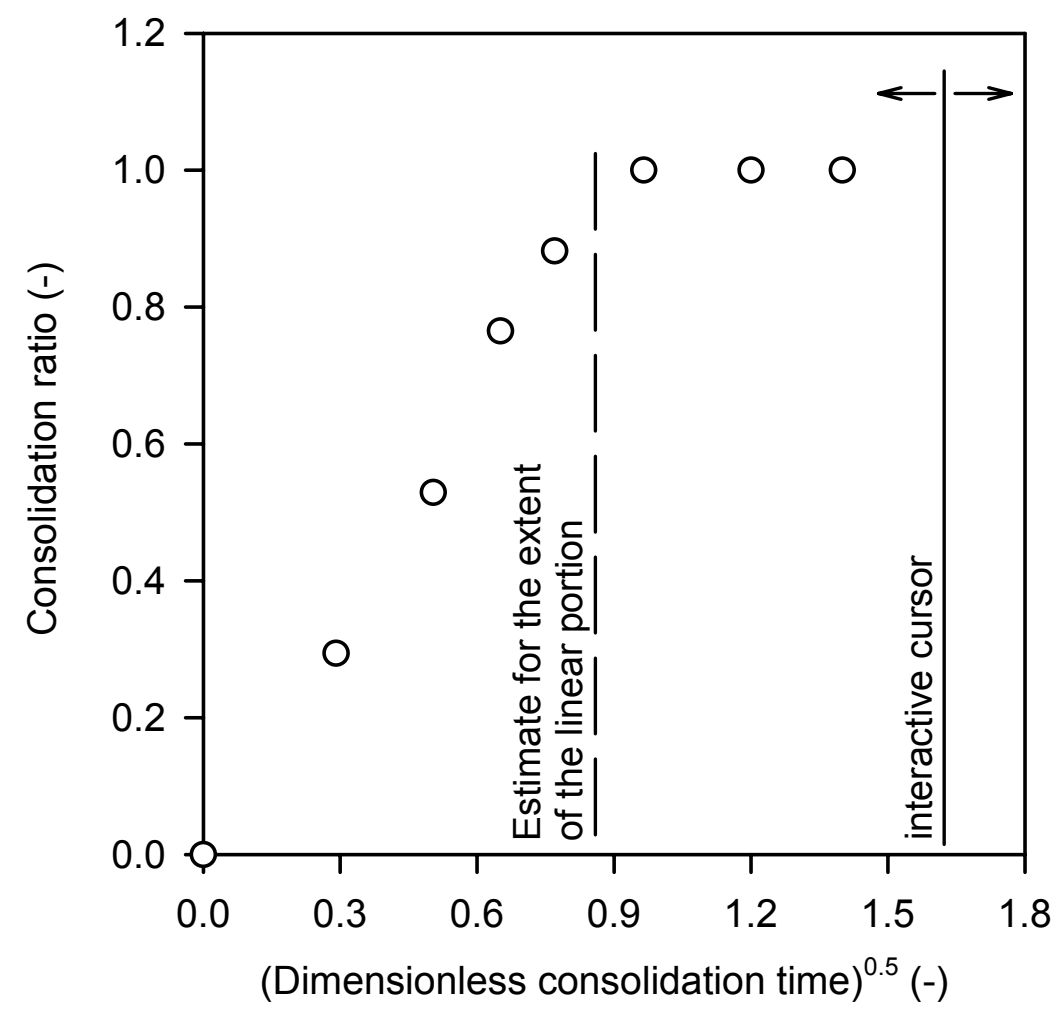

Figure 9: Plot used to identify the initial linear portion of a cake consolidation.

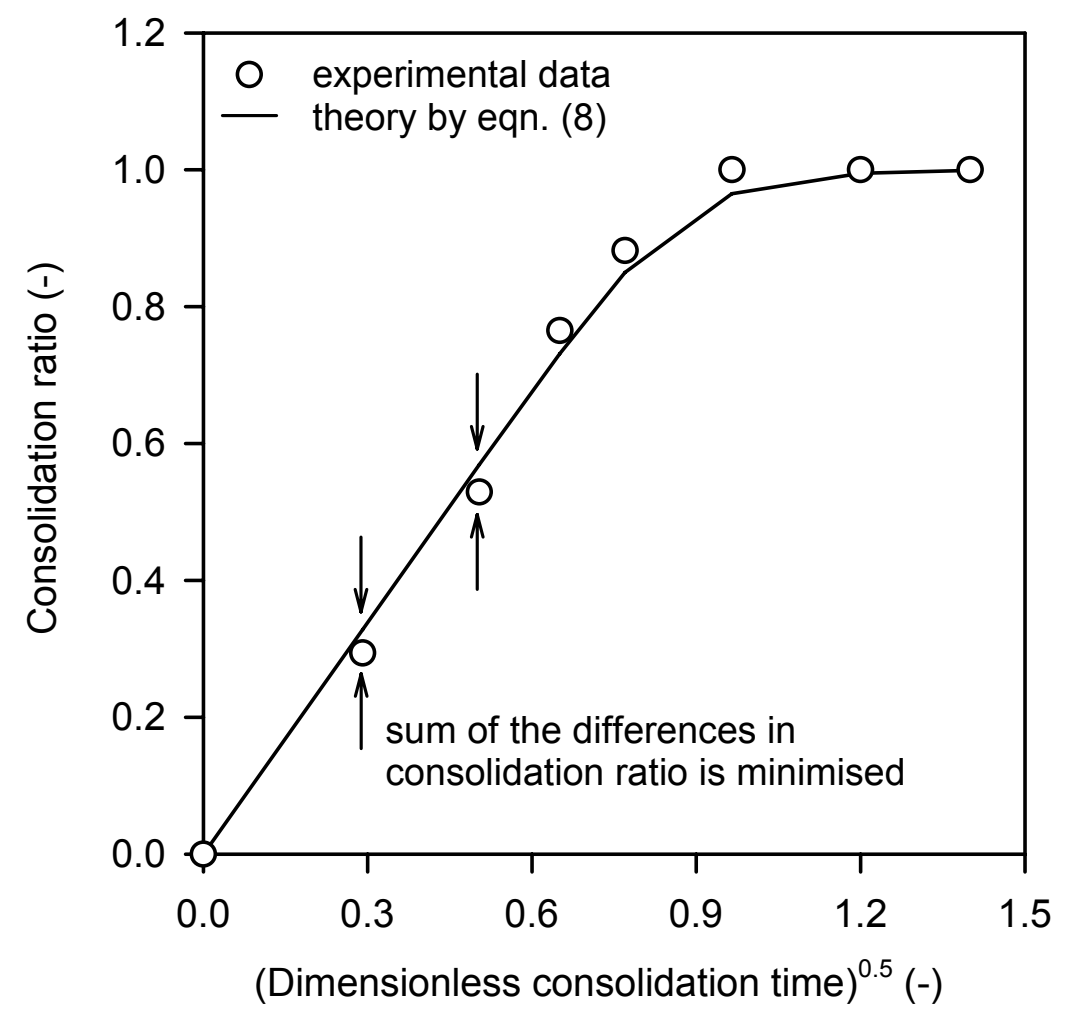

Figure 10: Plot used in the evaluation of the consolidation index. 


\begin{tabular}{|ll|c|c|c|c|}
\hline \multicolumn{2}{|l|}{ Experimental parameter } & Time $(\mathrm{s})$ & $\begin{array}{c}\text { Volume } \\
\left(\mathrm{cm}^{3}\right)\end{array}$ & Time $(\mathrm{s})$ & $\begin{array}{c}\text { Volume } \\
\left(\mathrm{cm}^{3}\right)\end{array}$ \\
\hline Piston diameter: & $0.043 \mathrm{~m}$ & 0 & 0 & 7200 & 129.4 \\
Press length: & $0.193 \mathrm{~m}$ & 180 & 16.0 & 9000 & 146.0 \\
Applied pressure: & $6.4 \mathrm{MPa}$ & 300 & 21.6 & 10800 & 160.5 \\
Solids density: & $2570 \mathrm{~kg} \mathrm{~m}^{-3}$ & 600 & 32.7 & 12600 & 175.0 \\
Fluid density: & $1000 \mathrm{~kg} \mathrm{~m}^{-3}$ & 720 & 36.0 & 14400 & 187.2 \\
Fluid viscosity: & $0.001 \mathrm{~Pa} \mathrm{~s}^{2}$ & 900 & 40.7 & 15000 & 191.5 \\
Feed conc.: & $0.145 \mathrm{v} / \mathrm{v}$ & 1080 & 45.2 & 15600 & 197.0 \\
& & 1200 & 48.3 & 16200 & 200.5 \\
& & 1380 & 52.2 & 16800 & 203.0 \\
& & 1500 & 54.4 & 17400 & 204.0 \\
& & 1800 & 60.0 & 18000 & 205.0 \\
& 2400 & 70.5 & 18600 & 205.5 \\
& & 3600 & 88.3 & 19800 & 206.0 \\
& & 5400 & 110.5 & 21600 & 206.0 \\
\hline
\end{tabular}

Table 1: Sample data from a typical piston press expression experiment. Initial mixture was an aqueous suspension of ground china clay $\left(x_{a v}=3.3 \mu \mathrm{m}, \mathrm{pH}=5.2\right)$.

\begin{tabular}{|ll|ll|}
\hline Filtration phase information & & \multicolumn{2}{l|}{ Consolidation phase information } \\
\hline Specific cake resistance: & $2.0 \times 10^{13} \mathrm{~m} \mathrm{~kg}^{-1}$ & Consolidation curve slope: & $2.8 \times 10^{-4} \mathrm{~s}^{-1}$ \\
Filter medium resistance: & $6.31 \times 10^{13} \mathrm{~m}^{-1}$ & Mod. consolidation coeff. & $1.7 \times 10^{-7} \mathrm{~m}^{2} \mathrm{~s}^{-1}$ \\
Effective feed concentration: & $517.7 \mathrm{~kg} \mathrm{~m}^{3}$ & Consolidation index: & 5.00 \\
Average cake formation rate: & $0.0197 \mathrm{~cm} \mathrm{~min}$ & Fit variance to consol. curve: & 0.00087 \\
Final cake porosity: & 0.482 & Final cake porosity: & 0.453 \\
Final cake voids ratio: & 0.932 & Final cake voids ratio: & 0.828 \\
Final cake moisture content: & $26.6 \%$ & Final cake moisture content: $24.3 \%$ \\
Final ratio mass wet/dry cake: & 1.36 & Final ratio mass wet/dry cake: 1.32 \\
Final mass dry cake/filter area: $71.9 \mathrm{~kg} \mathrm{~m}^{-2}$ & Final volume solids/filter area: $0.0280 \mathrm{~m}$ \\
\hline
\end{tabular}

Table 2: Results of analysis by software for the experimental data shown in Table 1. 


\begin{tabular}{|l|l|l|l|l|}
\hline Applied pressure $(\mathrm{MPa})$ & $\alpha_{a v}\left(\mathrm{~m} \mathrm{~kg}^{-1}\right)$ & $\left(e_{a v}\right)_{f}$ & $\left(e_{a v}\right)_{c}$ & $C_{c}\left(\mathrm{~m}^{2} \mathrm{~s}^{-1}\right)$ \\
\hline 0.33 & $5.5 \times 10^{12}$ & 1.53 & 1.35 & $3.0 \times 10^{-8}$ \\
1.65 & $1.1 \times 10^{13}$ & 1.20 & 1.08 & $7.1 \times 10^{-8}$ \\
2.36 & $1.3 \times 10^{13}$ & 1.13 & 1.04 & $1.1 \times 10^{-7}$ \\
6.41 & $2.0 \times 10^{13}$ & 0.93 & 0.83 & $1.7 \times 10^{-7}$ \\
10.79 & $3.3 \times 10^{13}$ & 0.81 & 0.71 & $1.4 \times 10^{-7}$ \\
14.50 & $3.2 \times 10^{13}$ & 0.74 & 0.65 & $2.2 \times 10^{-7}$ \\
20.56 & $3.8 \times 10^{13}$ & 0.68 & 0.57 & $2.3 \times 10^{-7}$ \\
Calculated scale-up & $\alpha_{0}(1-n)=8.91 \times 10^{12}$ & $\left(e_{0}\right)_{f}=1.30$ & $\left(e_{0}\right)_{c}=1.17$ & $C_{e 0}=5.75 \times 10^{-8}$ \\
coefficients & $n=0.48$ & $b_{f}=0.48$ & $b_{c}=0.44$ & $V=0.48$ \\
& 0.993 & 0.999 & 0.997 & 0.971 \\
Regression & & & & \\
correlation coefficient & & & & \\
\hline
\end{tabular}

Table 3: A typical example of the software evaluation of scale-up constants from experimental piston press data. Initial mixtures were aqueous suspensions of ground china clay $\left(x_{a v}=3.3 \mu \mathrm{m}\right.$, $\mathrm{pH}=5.2)$. 